\title{
Diagnostic Efficacy of Diffusion-Weighted Magnetic Resonance Imaging (DWI) in the Differentiation of Benign and Malignant Cervical Lymphadenopathies
}

\author{
Leila Aghaghazvini ${ }^{1,2}$, Bahman Rasuli ${ }^{2,{ }^{*}}$, Hashem Sharifian ${ }^{2,3}$, Fariba Hashemi ${ }^{1}$, Nasrin Yazdani ${ }^{4}$ and \\ Elahe Zaremehrjerdi ${ }^{5}$ \\ ${ }^{1}$ Department of Radiology, Shariati General Hospital, Tehran University of Medical Sciences, Tehran, Iran \\ ${ }^{2}$ Advanced Diagnostic and Interventional Radiology Research Center (ADIR), Imam Khomeini Hospital, Tehran University of Medical Sciences, Tehran, Iran \\ ${ }^{3}$ Department of Radiology, Amir Alam Hospital, Tehran University of Medical Sciences, Tehran, Iran \\ ${ }^{4}$ Otorhinolaryngology Research Center, Amir Alam Hospital, Tehran University of Medical Sciences, Tehran, Iran \\ ${ }^{5}$ Imam Khomeini Hospital, Medical Faculty of Tehran University of Medical Sciences, Tehran, Iran \\ "Corresponding author: Advanced Diagnostic and Interventional Radiology Research Center (ADIR), Imam Khomeini Hospital, Tehran University of Medical Sciences, Tehran, \\ Iran.Email: bahman_rasuli@yahoo.com
}

Received 2019 March 10; Revised 2020 April 09; Accepted 2020 April 20.

\begin{abstract}
Background: Pre-operative differentiation of benign from malignant cervical adenopathies remains a challenge. Several studies indicate parameters to discriminate between benign and malignant lymph nodes, which has not yet been enough.

Objectives: The aim of the current study was to assess the diagnostic efficacy of diffusion-weighted MR imaging (DWI) to differentiate benign from malignant cervical lymph nodes.

Patients and Methods: In all patients, we assessed axial and coronal fast spin echo T2 and T1-weighted images and T1-weighted after contrast injection. DWI sequences were implemented before contrast injection, in axial and coronal planes (b factor of 50, 500 and $1000 \mathrm{~s} / \mathrm{mm}^{2}$ ) and the apparent diffusion coefficient (ADC) maps were reconstructed. Data were assessed in mixed model analysis and results were compared with postoperative histopathologic findings.

Results: Thirty seven subjects were enrolled, 10 with benign lymphadenopathy and 27 patients with malignant lymphadenopathies before treatment. The mean ADC of the benign neck lymph nodes was $(1.00 \pm 0.34) \times 10^{-3} \mathrm{~mm}^{2} / \mathrm{s}$, while it was $(0.76 \pm 0.16) \times 10^{-3}$ $\mathrm{mm}^{2} / \mathrm{s}$ in malignant ones $(\mathrm{P}=0.058)$. The mean ADC of the metastatic nodes was $(0.81 \pm 0.14) \times 10^{-3} \mathrm{~mm}^{2} / \mathrm{s}$, while it was $(0.56 \pm$ $0.04) \times 10^{-3} \mathrm{~mm}^{2} / \mathrm{s}$ in lymphoma $(\mathrm{P}<0.001)$. The mean ADC of poorly differentiated metastatic nodes was significantly lower than that of good and moderately differentiated ones $\left[(0.86 \pm 0.13) \times 10^{-3} \mathrm{~mm}^{2} / \mathrm{s}\right.$ vs. $(0.66 \pm 0.02) \times 10^{-3} \mathrm{~mm}^{2} / \mathrm{s}$ respectively; $\left.\mathrm{P}=0.001\right]$. The area under the ROC curve of ADC was $0.69[95 \% \mathrm{CI}=0.52-0.83]$. Considering cut off points of $0.6 \times 10^{-3}, 0.95 \times 10^{-3}$, and $1.2 \times$ $10^{-3}$ yielded sensitivities were $15 \%, 92.5 \%$, and $100 \%$, respectively; while, in these cutoff points, specificities were $80 \%, 50 \%$, and $40 \%$, respectively.
\end{abstract}

Conclusion: DWI could be considered as an important diagnostic tool to differentiate enlarged cervical lymphadenopathies.

Keywords: Diffusion-Weighted Imaging (DWI), Neck, Lymphadenopathy, Lymphoma, Metastasis, Benign, Malignant

\section{Background}

The diagnosis of malignant cervical lymph nodes has an important role in the treatment of patients suffering from head and neck cancers and tumoral response. Different parameters utilized by conventional imaging methods include size, morphology, capsular breakthrough and abnormal internal structure.

Although the lymph node size is the most common criteria diagnosis, central necrosis in the lymph nodes is the most reliable sign of tumoral involvement (1). Several studies indicate that to discriminate between benign and malignant lymph nodes, the above parameters are not enough $(2,3)$. Intercellular water motion is analyzed with diffusion-weighted MR imaging (DWI). Every alteration in the water proton behavior results in a change in diffusionweighted sequence signal intensity consequently on apparent diffusion coefficient (ADC) maps (4). Our study was aimed to evaluate the potential diagnostic role of DWI in the discrimination between benign and malignant lymph nodes.

To evaluate the difference in molecular diffusion according to the structure and properties of the tissues, 
diffusion-weighted magnetic resonance imaging (DWI) is an effective well-established magnetic resonance imaging method.

The presence of any alternation in the water proton movement leads to a change in signal intensity in DWI results consequently on ADC maps (4).

This sequence is a helpful diagnostic tool for recognition between tumoral and non-tumoral tissues, especially in head and neck lymphadenopathies (5).

\section{Objectives}

The aim of this prospective study was to evaluate the diagnostic accurateness of DWI in differentiating benign from malignant cervical adenopathies.

\section{Patients and Methods}

\subsection{Patients}

In our prospective study, 37 subjects were included, of which 10 with benign lymphadenopathy and 27 patients with malignant lymphadenopathies underwent DWI imaging before the surgical procedure and histopathological examination.

The institutional Review Board and the local Ethics Committee at the university approved this study. After describing the study process in detail to the patients, written informed consent was obtained from all participants.

\subsection{MR Imaging Protocol}

All of the exams were done using a 3T GE 750w superconductive magnet scanner (GE Healthcare, Waukesha, WI, USA) by means of a head-neck standardized coil. The selected protocols were used in all cases as followed:

-Fast spin echo T2-weighted images(TR 5400 ms, TE 100 $\mathrm{ms}$, slice-thickness: $3 \mathrm{~mm}$ ) in the axial and coronal planes

- Fast spin echo T1-weighted images, with and without fat suppression (TR $700 \mathrm{~ms}$, TE $25 \mathrm{~ms}$, slice-thickness: 3 $\mathrm{mm}$ ) in the axial plane

- Fast spin echo fat saturation T1-weighted images, after administration of $25 \mathrm{~mL}$ of gadopentetate dimeglumine, in axial and coronal planes

- Single-shot echo planar diffusion sensitized sequences (DWI) (TR 3000 - 3500 ms/90 ms, slice thickness: $4 \mathrm{~mm}$ ) in the axial plane.

All of the mentioned protocols were found with a slice thickness of 3 - $4 \mathrm{~mm}$, a field of view (FOV) of $250 \mathrm{~mm}$, an intersection gap of $1 \mathrm{~mm}$, and a flip angle of $90^{\circ}$.

Before contrast administration, diffusion-weighted images were obtained in the axial plane. Utilizing the different b values $\left(50,500\right.$ and $\left.1000 \mathrm{~s} / \mathrm{mm}^{2}\right)$, the diffusionsensitizing gradient was applied in all three orthogonal planes (X, Y, Z). Using MRI machine software, apparent diffusion coefficient (ADC) maps were also calculated automatically and added to the sequences.

\subsection{Image Interpretation and Analysis}

Blind interpretation of the images was done with no access to the histopathological report of the nodes. To assess the nodes, the conventional $\mathrm{T} 1$ and $\mathrm{T} 2$-weighted sequences were performed. To calculate the ADC values, ADC maps derived from DWI images were utilized and ADC values were determined automatically by the software as well. The largest abnormal node containing areas of homogeneous enhancement was chosen in every patient case for the investigation. Drawing a region of interest (ROI) covering as much as possible of the involved lymph nodes, the ADC values were measured (Figure 1). The average size of the region of interest measured in the pathological lymph nodes was $589 \mathrm{~mm}^{2}$ (minimum, $196 \mathrm{~mm}^{2}$ : maximum, 982 $\mathrm{mm}^{2}$ ).

To prevent a false high ADC caused by the low count of tumoral cells, we also excluded the necrotic areas of the lymph nodes (1).

In each case, the findings obtained from the measurements on MRI were compared with the histopathological results. After surgery, the topographic correlation of the involved lymph nodes was performed to assure that the dissected nodes are compatible with the same demarcated at

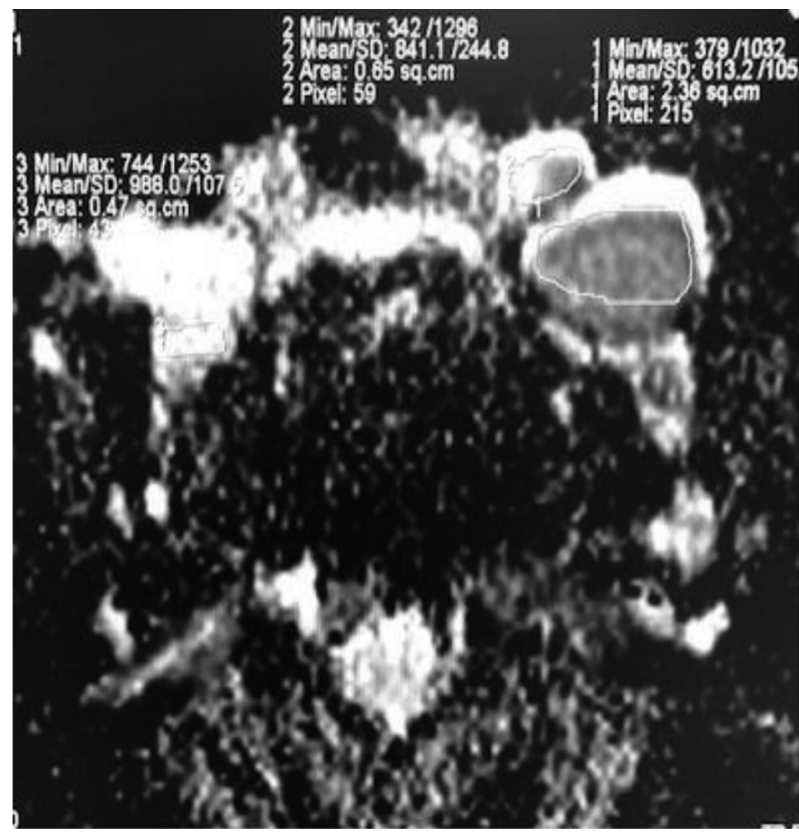

Figure 1. Region of interest (ROI) utilization at apparent diffusion coefficient (ADC) map to estimate $\mathrm{ADC}$ values 
the MRI system. Short axis diameter, exact location, and association to the adjacent anatomical structures are considered as topographic findings.

\subsection{Statistical Data Analysis}

The patients were classified into benign, metastasis and lymphoma, considering the histopathological results of the existing lymph nodes. Using SPSS (IBM SPSS Statistics, Version 21.0. Armonk, NY: IBM Corp.) V. 21, statistical analysis was calculated.

In the three groups (benign, metastatic and lymphoma), the mean ADC values with standard deviations were measured and compared statistically using Post Hoc test with ANOVA (one-way analysis of variance) to compare the ADC mean values among three different histological types. In addition, metastasis was subdivided into good-moderate and poorly differentiated categories. To assess the difference in ADC values between these three mentioned groups, $t$-test was used.

To compare the diagnostic certainty of the value of diffusion-weighted MR imaging between benign versus malignant (metastasis and lymphoma) adenopathy, we utilized the receiver operating characteristic (ROC) curve as a graphical plot with estimation of the area under the curve (AUC). We also used several cut off points of ADC values to reach the best threshold by means of Kappa test for the differentiation of benign versus malignant nodes. A value of $\mathrm{P}<0.05$ was accepted significant as well.

\section{Results}

The recent prospective study included thirty-seven patients with suspicious malignant cervical nodes who underwent MRI including DWI sequences and followed by histopathological examination after either surgery or neck dissection. Regarding histopathological findings, lymphadenopathy was divided into benign cervical nodes (lymphadenitis) in 10 cases, metastasis from the head and neck cancer in 22 cases (sixteen cases reclassified as good and moderately differentiated and six cases with poorly differentiated cancers) and lymphoma (non-Hodgkin lymphoma) in five cases (Tables 1 and 2). The mean short-axis diameter of selected lymph nodes was $22.3 \mathrm{~mm}$ (range $=11$ - $42 \mathrm{~mm}$ ).

The mean ADC values of the benign, metastasis (goodmoderately differentiated and poorly differentiated metastasis) and lymphoma groups were $1.00 \pm 0.34 \times 10^{-3}$, $0.81 \pm 0.14 \times 10^{-3}\left(0.86 \pm 0.13 \times 10^{-3}\right.$ for good-moderately differentiated and they were $0.66 \pm 0.02 \times 10^{-3}$ for poorly differentiated metastasis) and $0.56 \pm 0.02 \times 10^{-3}$, sequentially (Table 2 and Figure 2).

Using post hoc analysis and one-way analysis of variance (ANOVA) it was indicated that the ADC values of the

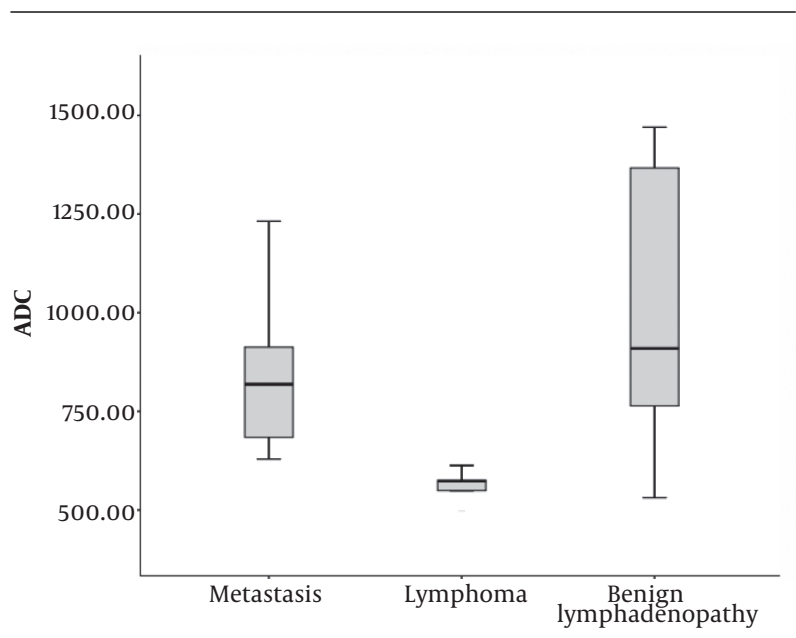

Figure 2. Box schemes of the apparent diffusion coefficient (ADC) values of benign lymphadenopathy, nodal lymphoma, and metastatic nodes

benign cervical nodes were markedly higher compared to the poorly differentiated metastatic nodes (Figure 3) and the nodes involved by lymphoma (Figure 4$)(\mathrm{P}<0.001)$. The ADC values of the good and moderately differentiated metastatic lymph nodes were notably higher compared to the nodal lymphoma $(\mathrm{P}=0.02)$.

The mean ADC values of benign lymph nodes $1.00 \pm$ $0.34 \times 10^{-3} \mathrm{~mm}^{2} / \mathrm{s}$ were higher compared to the mean ADC values of malignant lymph nodes (metastatic and lymphoma) $0.76 \pm 0.16 \times 10^{-3} \mathrm{~mm}^{2} / \mathrm{s}(t=2.12, \mathrm{P}=0.058)$.

Compared to the mean ADC value of metastatic nodes $0.81 \pm 0.14 \times 10^{-3} \mathrm{~mm}^{2} / \mathrm{s}$, the mean ADC of non-Hodgkin lymphoma $0.56 \pm 0.04 \times 10^{-3} \mathrm{~mm}^{2} / \mathrm{s}$ was lower and there was a meaningful difference between the ADC values between these two categories $(t=3.76, \mathrm{P}<0.001)$ (Table 3 ).

To investigate the ADC value capability in the differentiation between benign and malignant (metastasis and lymphoma) lymph nodes (Figure 5), the receiver operating characteristic (ROC) curve is utilized as a diagnostic method. The estimated area under the ROC curve was 0.69 with a confidence interval of $0.52-0.83$, standard error $=$ 0.12 and $\mathrm{P}>0.05$.

The ADC value cut off for distinction between benign and malignant lymph nodes was $0.95 \times 10^{-3} \mathrm{~mm}^{2} / \mathrm{s}$ with a sensitivity of $92.5 \%$, specificity of $50 \%$, positive predictive value of $83.3 \%$, negative predictive value of $71.3 \%$, kappa test of $20.6 \%$, and $P$ value of 0.1 .

In addition, with the considering cut off points of $0.6 \times$ $10^{-3}$ and $1.2 \times 10^{-3} \mathrm{~mm}^{2} / \mathrm{s}$, moreover, the $0.95 \times 10^{-3} \mathrm{~mm}^{2} / \mathrm{s}$ yielded sensitivities of $15 \%$ and $100 \%$, respectively while, in these cutoff points, specificities were $80 \%$ and $40 \%$, respectively. 


\begin{tabular}{|c|c|c|c|c|}
\hline Group/patient No. & Age $(y)$ & Sex & Histopathologic diagnosis & $\begin{array}{l}\text { ADC value }\left(\times 1^{-3}\right. \\
\left.\mathbf{m m}^{2} / \mathbf{s}\right)\end{array}$ \\
\hline \multicolumn{5}{|l|}{ Lymphadenitis } \\
\hline 1 & 36 & M & Chronic inflammation and fibrosis & 1.235 \\
\hline 2 & 42 & M & Chronic inflammation and fibrosis & 0.863 \\
\hline 3 & 56 & M & Active chronic inflammation and fibrosis & 1.118 \\
\hline 4 & 48 & M & Active chronic inflammation and fibrosis & 0.954 \\
\hline 5 & 41 & M & Chronic inflammation and fibrosis & 1.366 \\
\hline 6 & 37 & $\mathrm{~F}$ & Chronic inflammation, giant cell reaction and fibrosis & 0.863 \\
\hline 7 & 23 & $\mathrm{~F}$ & Active chronic inflammation and fibrosis & 0.852 \\
\hline 8 & 32 & $\mathrm{~F}$ & Chronic inflammation and fibrosis & 0.601 \\
\hline 9 & 36 & $\mathrm{~F}$ & Active chronic inflammation and fibrosis & 0.531 \\
\hline 10 & 47 & M & Chronic inflammation and fibrosis & 1.310 \\
\hline \multicolumn{5}{|l|}{ Metastasis } \\
\hline 1 & 61 & M & Adenocarcinoma, good and moderately differentiated & 0.702 \\
\hline 2 & 64 & M & Adenocarcinoma, good and moderately differentiated & 0.720 \\
\hline 3 & 51 & M & Adenocarcinoma, good and moderately differentiated & 0.864 \\
\hline 4 & 58 & M & Adenocarcinoma, good and moderately differentiated & 0.822 \\
\hline 5 & 42 & $\mathrm{~F}$ & Adenocarcinoma, good and moderately differentiated & 1.231 \\
\hline 6 & 43 & $\mathrm{~F}$ & Adenocarcinoma, good and moderately differentiated & 0.871 \\
\hline 7 & 29 & M & Laryngeal SCC, poorly differentiated & 0.631 \\
\hline 8 & 28 & $\mathrm{~F}$ & PTC, good and moderately differentiated & 0.897 \\
\hline 9 & 43 & M & Adenocarcinoma, poorly differentiated & 0.613 \\
\hline 10 & 42 & M & Laryngeal SCC, poorly differentiated & 0.688 \\
\hline 11 & 51 & M & Laryngeal SCC, poorly differentiated & 0.695 \\
\hline 12 & 46 & M & Mucoepidermoid carcinoma, good and moderately differentiated & 0.673 \\
\hline 13 & 58 & $\mathrm{~F}$ & PTC, good and moderately differentiated & 0.773 \\
\hline 14 & 57 & M & $\begin{array}{l}\text { Carcinoma ex pleomorphic adenoma, good and moderately } \\
\text { differentiated }\end{array}$ & 0.858 \\
\hline 15 & 57 & M & SCC, NPC undifferentiated type & 0.690 \\
\hline 16 & 64 & M & SCC, NPC moderately differentiated type & 0.913 \\
\hline 17 & 64 & M & SCC, NPC moderately differentiated type & 0.820 \\
\hline 18 & 65 & M & Laryngeal SCC, moderately differentiated type & 0.919 \\
\hline 19 & 56 & M & SCC, NPC moderately differentiated type & 0.969 \\
\hline 20 & 71 & M & Laryngeal SCC, moderately differentiated type & 0.961 \\
\hline 21 & 68 & M & SCC, NPC undifferentiated type & 0.662 \\
\hline 22 & 73 & M & Prostate carcinoma, good and moderately differentiated type & 0.930 \\
\hline \multicolumn{5}{|l|}{ Lymphoma } \\
\hline 1 & 64 & $\mathrm{~F}$ & B cell lymphoma & 0.575 \\
\hline 2 & 46 & $\mathrm{~F}$ & B cell lymphoma & 0.573 \\
\hline 3 & 37 & $\mathrm{~F}$ & Diffuse large B-cell lymphoma & 0.650 \\
\hline 4 & 51 & $\mathrm{~F}$ & T-cell lymphoblastic type lymphoma & 0.496 \\
\hline 5 & 44 & M & B cell lymphoma & 0.549 \\
\hline
\end{tabular}

Abbreviations: ADC, apparent diffusion coefficient; F, female; M, male; NPC, nasopharyngeal carcinoma; PTC, papillary thyroid carcinoma; SCC, squamous cell carcinoma

\section{Discussion}

Existing lymph node metastases is a key prognostic factor not only for the staging of malignant disease but it also has a significant impact on the treatment result in patients suffering from head and neck cancer $(6,7)$. Ultrasound, MRI and contrast-enhanced computed tomogra- 


\begin{tabular}{|c|c|c|}
\hline & Number of cases & ADC values (mean $\pm \mathrm{SD})\left(\mathrm{mm}^{2} / \mathbf{s}\right)$ \\
\hline Benign & 10 & $1.00 \pm 0.34 \times 10^{-3}$ \\
\hline Metastasis & 22 & $0.81 \pm 0.14 \times 10^{-3}$ \\
\hline Good and moderately-differentiated & 16 & $0.86 \pm 0.13 \times 10^{-3}$ \\
\hline Poorly-differentiated & 6 & $0.66 \pm 0.02 \times 10^{-3}$ \\
\hline Non-Hodgkin lymphoma & 5 & $0.56 \pm 0.04 \times 10^{-3}$ \\
\hline
\end{tabular}

Abbreviations: ADC, apparent diffusion coefficient; SD, standard deviation
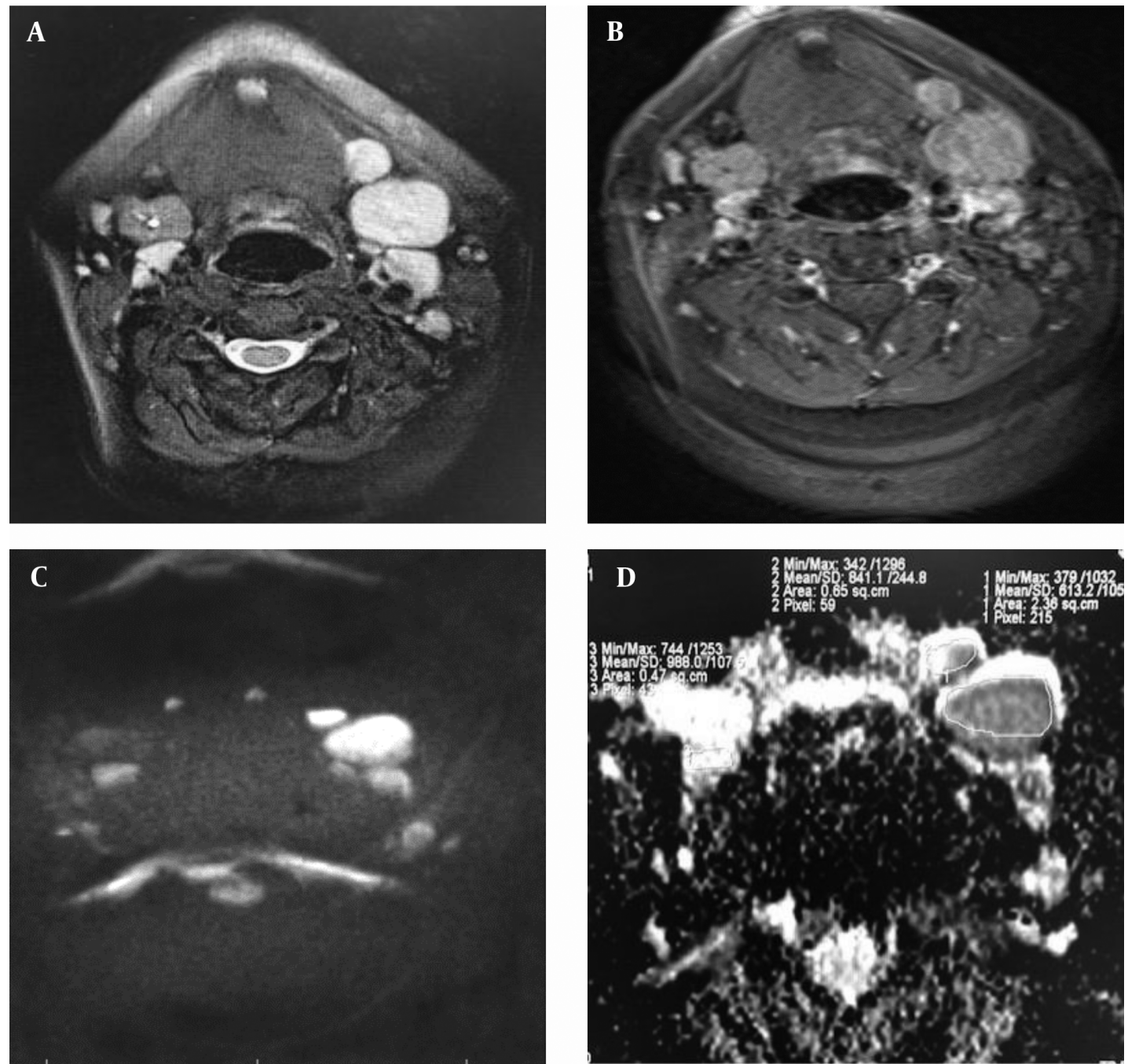

Figure 3. Patient with resection of left submandibular gland infiltrated by adenocarcinoma and poorly-differentiated metastatic nodes: axial T2 (A) and post-contrast T1 (B) sequences with fat suppression show enlarged cervical lymph nodes with enhancement at the bed of the resected left submandibular gland. At diffusion-weighted imaging (DWI) (C) with a high b value of 1000 , the nodes show water restriction with low signal intensity on the corresponding apparent diffusion coefficient (ADC) map (D) (ADC value of $0.61 \times 10^{-3} \mathrm{~mm}^{2} / \mathrm{s}$ ). Next to the bed of right submandibular gland, there is a reactive lymph node with a DWI slightly hyperintensity as well as high signal intensity on the corresponding ADC map (D) (ADC value of $0.98 \times 10^{-3} \mathrm{~mm}^{2} / \mathrm{s}$ ).

phy are helpful modalities to detect the enlarged cervical lymph nodes; however, these methods do not provide the 

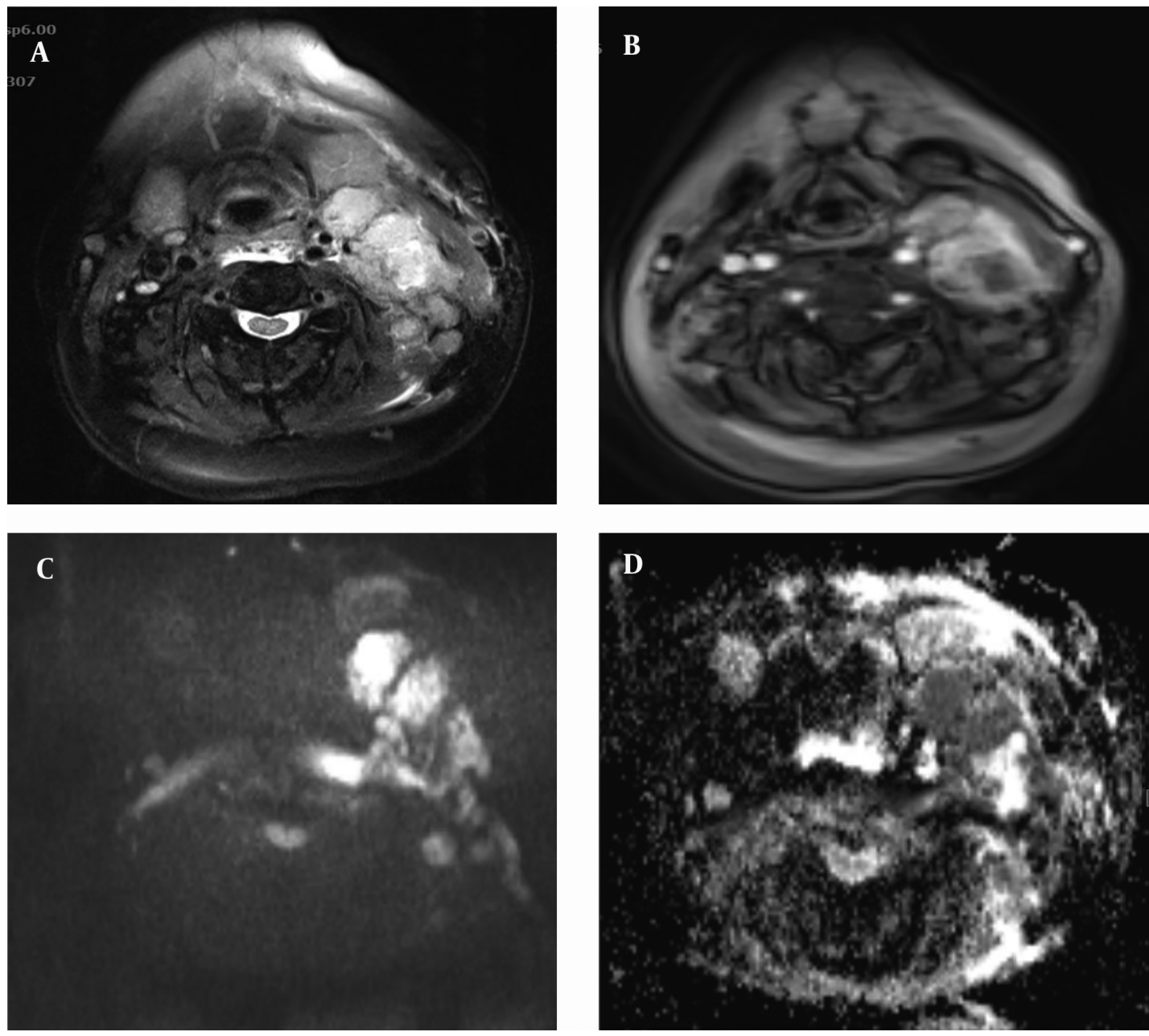

Figure 4. Patient with non-Hodgkin lymphoma: axial T2 (A) and post-contrast T1 (B) sequences with fat suppression show enlarged cervical lymph nodes at the left cervica chain. The nodes are hyperintense at diffusion-weighted imaging (DWI) b 1000 (C) and low signal intensity on the corresponding apparent diffusion coefficient (ADC) map (D) (ADC value of $0.54 \times 10^{-3} \mathrm{~mm}^{2} / \mathrm{s}$ ).

\begin{tabular}{lccc}
\hline Table 3. Comparison of $t$-test and P Value Between ADC Values in Various Kinds of Neck Adenopathy & \\
\hline & Benign versus malignant & Metastasis versus non-Hodgkin lymphoma & $\begin{array}{c}\text { Good and moderately-differentiated versus poorly-differentiated } \\
\text { metastasis }\end{array}$ \\
\hline $\boldsymbol{t}$-test & 2.12 & 3.76 & 3.76 \\
P value & 0.058 & $<0.001$ & 0.001 \\
\hline
\end{tabular}

ideal diagnostic accuracy $(4,8)$. Single photon emission CT (SPECT) and positron emission tomography (PET) provide us the functional information. However, they are expensive, invasive, have less availability and relatively low spatial resolution (9-11).

Recently, magnetic resonance with diffusion-weighted imaging is used which could improve the diagnostic accuracy in the differentiation of benign and malignant lymph nodes $(12,13)$.
Diffusion-weighted imaging is a noninvasive technique by which the diffusion of water protons is measured in the extracellular and intracellular spaces through cell membranes. Therefore, the presence of any changes in tissue cell architecture, including the number of extracellular versus intracellular water protons, will change the diffusion coefficient of the tissue cells (13).

Thus, DWI could show details of biological behavior of tissue cells and had a diagnostic role in the differentiation 


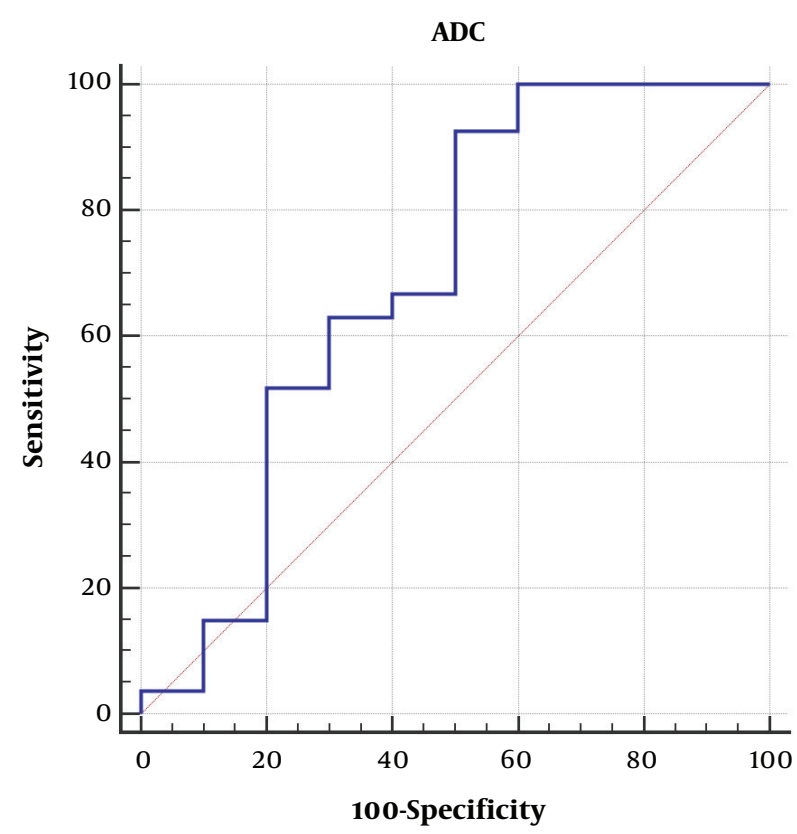

Figure 5. Differentiation between benign and malignant (metastatic and lymphoma) lymph nodes by the ROC curve of apparent diffusion coefficient(ADC) value. The calculated area under the curve (AUC) is 0.69 .

between benign and malignant tumors with the gradual reduction in ADC values from the benign lesions toward the malignant tumors. Moreover, a significant relationship exists between the ADC values and tumor cellularity (14).

Several studies show that metastatic lymph nodes present a diffusivity reduction, which could be caused by tumoral tissue hypercellularity leading to a raised nucleuscytoplasm ratio and perfusion (13). However, in cases of lymphoma regarding high compact cellularity and decreased extracellular space, diffusion-weighted imaging has a key role.

Only a few studies have examined diffusion-weighted MR imaging on the characterization of head and neck lesions $(5,13,15-17)$.

In the recent study, 37 subjects with distended neck lymph nodes, ten patients suffering from benign lymphadenopathy, 22 patients suffering from head and neck cancer metastasis and five patients with nodal lymphoma were included.

The mean ADC values of benign, metastasis (goodmoderately differentiated and poorly differentiated metastasis) and lymphoma groups were $1.00 \pm 0.34 \times 10^{-3}$ $\mathrm{mm}^{2} / \mathrm{s}, 0.81 \pm 0.14 \times 10^{-3} \mathrm{~mm}^{2} / \mathrm{s}\left(0.86 \pm 0.13 \times 10^{-3} \mathrm{~mm}^{2} / \mathrm{s}\right.$ for good-moderately differentiated and $0.66 \pm 0.02 \times 10^{-3}$ $\mathrm{mm}^{2} / \mathrm{s}$ for poorly differentiated metastasis) and $0.56 \pm$ $0.04 \times 10^{-3} \mathrm{~mm}^{2} / \mathrm{s}$, respectively.
The mean ADC values of malignant neck lymph nodes (metastatic nodes and nodal lymphoma) were significantly lower compared to benign lymph nodes $(\mathrm{P}=0.058)$. Additionally, ADC values of nodal lymphoma were significantly lower in comparison with the metastatic nodes $(\mathrm{P}<$ 0.001). This is similar to several earlier studies $(5,12,17-19)$.

Abdel Razek et al. (5) showed that the mean ADC value of benign cervical lymph nodes $\left(1.64 \pm 0.16 \times 10^{-3} \mathrm{~mm}^{2} / \mathrm{s}\right)$ was considerably higher compared to the metastasis (1.09 $\left.\pm 0.11 \times 10^{-3} \mathrm{~mm}^{2} / \mathrm{s}\right)$ and lymphomatous $(0.97 \pm 0.27 \times$ $\left.10-3 \mathrm{~mm}^{2} / \mathrm{s}\right)$ groups $(\mathrm{P}<0.04)$. Perrone et al. (18) indicated that compared to the mean ADC values of metastasis and lymphomatous groups $\left(0.85 \times 10^{-3} \mathrm{~mm}^{2} / \mathrm{s}\right)$, the mean ADC value of benign lymph nodes $\left(1.448 \times 10^{-3} \mathrm{~mm}^{2} / \mathrm{s}\right)$ was significantly higher $(\mathrm{P}<0.01)$. de Bondt et al. (19) reported that ADC values of benign lymph nodes were notably higher than that of malignant groups with mean values of $1.2 \pm 0.24 \times 10^{-3} \mathrm{~mm}^{2} / \mathrm{s}$ and $0.85 \pm 0.19 \times 10^{-3} \mathrm{~mm}^{2} / \mathrm{s}$, respectively.

King et al. (17) studied the malignant neck lymphadenopathy and revealed that the ADC value of metastatic lymph nodes in squamous cell carcinoma was markedly higher in comparison with the ADC value of nodal lymphoma.

Furthermore, Sumi et al. (12) reported the lowest ADC values for lymphoma and the highest values for metastatic nodes. Moreover, they also showed that benign lymphadenopathy had high levels of ADC values in relation to nodal lymphomas $(\mathrm{P}<0.05)$. On the other hand, compared to metastatic nodes $\left(0.410 \pm 0.105 \times 10^{-3}\right.$ $\left.\mathrm{mm}^{2} / \mathrm{s}\right)$, ADC values of inflammatory nodes $(0.302 \pm 0.062$ $\left.\times 10^{-3} \mathrm{~mm}^{2} / \mathrm{s}\right)$ were significantly lower $(\mathrm{P}<0.01)$. They understood that the main reason for higher ADC values in metastatic nodes compared to benign nodes could be due to the presence of central necrosis in $48 \%$ of their metastatic lymph nodes leading to the large variability in the metastatic nodes ADC value.

The mean ADC values of the good and moderately differentiated metastasis in our study $\left(0.86 \pm 0.13 \times 10^{-3}\right.$ $\mathrm{mm}^{2} / \mathrm{s}$ ) was considerably higher than the mean ADC value of poorly differentiated metastasis $\left(0.66 \pm 0.23 \times 10^{-3}\right.$ $\left.\mathrm{mm}^{2} / \mathrm{s}\right)(\mathrm{P}=0.001)$. This is inconsistent with the similar previous study (15) which indicated that the mean ADC values of the good and moderately differentiated metastatic lymph nodes $\left(1.13 \pm 0.11 \times 10^{-3} \mathrm{~mm}^{2} / \mathrm{s}\right)$ were considerably higher in comparison to the mean ADC values of poorly ifferentiated metastatic groups $\left(0.89 \pm 0.12 \times 10^{-3} \mathrm{~mm}^{2} / \mathrm{s}\right)(\mathrm{P}$ $<0.02$ ).

This is also similar to the study conducted by Sumi et al. (15) which explained that hypercellularity and high nucleus-cytoplasm ratio in poorly differentiated carcinoma resulted in decreased extracellular matrix and decreased diffusion space of water protons in the intracellu- 
lar and extracellular dimensions.

Considering nodal lymphoma, the mean ADC of nonHodgkin lymphoma $\left(0.56 \pm 0.04 \times 10^{-3} \mathrm{~mm}^{2} / \mathrm{s}\right)$ was considerably lower than the mean ADC of metastatic lymph nodes $\left(0.81 \pm 0.14 \times 10^{-3} \mathrm{~mm}^{2} / \mathrm{s}\right)(t=3.76, \mathrm{P}<0.001)$.

This is compatible with the results of Perrone et al. (18) who verified the reduced ADC value in nodal lymphoma related to the increased cellularity and the decreased extracellular space.

For differentiation between benign and malignant nodes, the best ADC threshold value was $0.95 \times 10^{-3} \mathrm{~mm}^{2} / \mathrm{s}$ with a sensitivity, specificity and $\mathrm{P}$ value of $92.5 \%, 50 \%$ and 0.1 , respectively. The receiver operating characteristic (ROC) curve was utilized for discriminating benign from malignant lymph nodes based on the calculated area under the curve of 0.69 in the study $(\mathrm{P}>0.05)$.

In a study conducted by Perrone et al. (18), the optimal threshold value for the diagnosis of malignant cervical lymph nodes was $1.03 \times 10^{-3} \mathrm{~mm}^{2} / \mathrm{s}$, with a $100 \%$ sensitivity, 92.9\% specificity, and ROC curve of 0.983 .

Abdel Razek et al. (5) indicated the mean ADC value of $1.38 \times 10^{-3} \mathrm{~mm}^{2} / \mathrm{s}$ as a threshold value for differentiating benign from malignant lymph nodes, with an accuracy of $96 \%$, specificity of $88 \%$, sensitivity of $98 \%$, and ROC curve of 0.955 .

In addition, de Bondt et al. (19) indicated the optimum ADC threshold value of $1.0 \times 10^{-3} \mathrm{~mm}^{2} / \mathrm{s}$ for differentiating benign from malignant nodes with a sensitivity and specificity of $92.3 \%$ and $83.9 \%$, respectively.

In the recent study, considering the fact that strong susceptibility artifacts created by adjacent bony structures of the backbone led to the increased variability in ADC value estimations, no spine was used as proper reference tissue for ADC normalization purposes (20).

We utilized the sternocleidomastoid muscle with the mean ADC value of $1.410 \pm 0.105 \times 10^{-3} \mathrm{~mm}^{2} / \mathrm{s}$ as reference tissue for ADC normalization.

One of the restrictions of the current study was the use of high b value in the smallest lymph causing the reduced signal to noise ratio that hampered ADC measurements. Another constraint was the small cohort study because the statistical tests were conducted on the number of patients regardless of the number of lymphadenopathies, to prevent the confounding influence of multiple nodes per patient on the outcome.

In conclusion, the mean $\mathrm{ADC}$ value of metastasis (goodmoderately differentiated and poorly differentiated) is less compared to the mean ADC values of benign swollen neck lymph nodes. The mean ADC value of poorly differentiated metastasis is lower in comparison with the mean ADC value of good and moderately differentiated groups. The mean ADC value of non- Hodgkin lymphoma is lower than the benign and metastatic lymph nodes.
For distinguishing malignant from benign lymph nodes, the optimal threshold is $0.95 \times 10^{-3} \mathrm{~mm}^{2} / \mathrm{s}$. Notwithstanding future advances and improvements are still to be anticipated, nevertheless, DWI as a non-invasive practical imaging modality could play an important role in the differentiation of benign from malignant lymph nodes.

\section{Acknowledgments}

The authors hereby acknowledge the staff of the Radiology Center of Imam Khomeini and Amir Alam hospitals, Tehran, for their kindness.

\section{Footnotes}

Authors' Contributions: Bahman Rasuli observed the design and data collection and the procedures for each case. Elahe Zaremehrjerdi prepared the draft of the manuscript and contributed to data collection. Fariba Hashemi contributed to data collection. Leila Aghaghazvini was in charge of statistics and manuscript revisions and Nasrin Yazdani observed the procedure for each case.

Conflict of Interests: We have no conflicts of interest to declare.

Ethical Approval: IR.TUMS.REC.1394.1808.

Funding/Support: This study was performed under financial support from Tehran University of Medical Sciences.

Patient Consent: The Institutional Review Board and the local Ethics Committee at the university approved this study. After describing the study process in detail to the patients, written informed consent was obtained from all participants.

\section{References}

1. King AD, Tse GM, Ahuja AT, Yuen EH, Vlantis AC, To EW, et al. Necrosis in metastatic neck nodes: diagnostic accuracy of CT, MR imaging, and US. Radiology. 2004;230(3):720-6. doi: 10.1148/radiol.2303030157. [PubMed: 14990838].

2. van den Brekel MW, Castelijns JA, Snow GB. The size of lymph nodes in the neck on sonograms as a radiologic criterion for metastasis: how reliable is it? AJNRAm J Neuroradiol.1998;19(4):695-700. [PubMed: 9576657].

3. van den Brekel MW, Castelijns JA, Snow GB. Detection of lymph node metastases in the neck: radiologic criteria. Radiology.1994;192(3):6178. doi: 10.1148/radiology.192.3.8058923. [PubMed: 8058923].

4. Rowley HA, Grant PE, Roberts TP. Diffusion MR imaging. Theory and applications. Neuroimaging Clin NAm.1999;9(2):343-61. [PubMed: 10318719].

5. Abdel Razek AA, Soliman NY, Elkhamary S, Alsharaway MK, Tawfik A. Role of diffusion-weighted MR imaging in cervical lymphadenopathy. Eur Radiol. 2006;16(7):1468-77. doi: 10.1007/s00330-005-0133-x. [PubMed: 16557366]. 
6. Kalnins IK, Leonard AG, Sako K, Razack MS, Shedd DP. Correlation between prognosis and degree of lymph node involvement in carcinoma of the oral cavity. Am JSurg.1977;134(4):450-4. doi: 10.1016/00029610(77)90376-2. [PubMed: 911028].

7. Jakobsen J, Hansen O, Jorgensen KE, Bastholt L. Lymph node metastases from laryngeal and pharyngeal carcinomas-calculation of burden of metastasis and its impact on prognosis. Acta Oncol. 1998;37(5):489-93. doi:10.1080/028418698430467. [PubMed: 9831380].

8. Curtin HD, Ishwaran H, Mancuso AA, Dalley RW, Caudry DJ, McNeil BJ. Comparison of CT and MR imaging in staging of neck metastases. Radiology. 1998;207(1):123-30. doi: 10.1148/radiology.207.1.9530307. [PubMed: 9530307].

9. Yamazaki Y, Saitoh M, Notani K, Tei K, Totsuka Y, Takinami S, et al. Assessment of cervical lymph node metastases using FDG-PET in patients with head and neck cancer. Ann Nucl Med. 2008;22(3):177-84. doi:10.1007/s12149-007-0097-9. [PubMed: 18498032].

10. Jeong HS, Baek CH, Son YI, Ki Chung M, Kyung Lee D, Young Choi J, et al. Use of integrated 18F-FDG PET/CT to improve the accuracy of initial cervical nodal evaluation in patients with head and neck squamous cell carcinoma. Head Neck. 2007;29(3):203-10. doi:10.1002/hed.20504 [PubMed: 17111430].

11. Kotani J, Kawabe J, Higashiyama S, Kawamura E, Oe A, Hayashi T, et al. Evaluation of diagnostic abilities of Ga-SPECT for head and neck lesions. Ann Nucl Med. 2008;22(4):297-300. doi: 10.1007/s12149-007-0116x. [PubMed: 18535880].

12. Sumi M, Van Cauteren M, Nakamura T. MR microimaging of benign and malignant nodes in the neck. AJR Am J Roentgenol. 2006;186(3):749-57. doi: 10.2214/AJR.04.1832. [PubMed: 16498102].

13. Wang J, Takashima S, Takayama F, Kawakami S, Saito A, Matsushita $T$, et al. Head and neck lesions: characterization with diffusionweighted echo-planar MR imaging. Radiology. 2001;220(3):621-30. doi: 10.1148/radiol.2202010063. [PubMed: 11526259].

14. Belli P, Costantini M, Bufi E, Magistrelli A, La Torre G, Bonomo L. Diffusion-weighted imaging in breast lesion evaluation. Radiol Med. 2010;115(1):51-69. doi: 10.1007/s11547-009-0430-6. [PubMed: 19902330].

15. Sumi M, Sakihama N, Sumi T, Morikawa M, Uetani M, Kabasawa H, et al. Discrimination of metastatic cervical lymph nodes with diffusionweighted MR imaging in patients with head and neck cancer.AJNRAm J Neuroradiol. 2003;24(8):1627-34. [PubMed:13679283].

16. Herneth AM, Czerny C, Krestan C. Role of diffusion-weighted MRI in the characterization of lymph node metastasis. XVI International Congress of Head and Neck Radiology. 2003.

17. King AD, Ahuja AT, Yeung DK, Fong DK, Lee YY, Lei KI, et al. Malignant cervical lymphadenopathy: diagnostic accuracy of diffusionweighted MR imaging. Radiology. 2007;245(3):806-13. doi: 10.1148/radiol.2451061804. [PubMed: 17911539].

18. Perrone A, Guerrisi P, Izzo L, D’Angeli I, Sassi S, Mele LL, et al. Diffusion-weighted MRI in cervical lymph nodes: differentiation between benign and malignant lesions. EurJ Radiol. 2011;77(2):281-6. doi: 10.1016/j.ejrad.2009.07.039. [PubMed: 19716671].

19. de Bondt RB, Hoeberigs MC, Nelemans PJ, Deserno WM, PeutzKootstra C, Kremer B, et al. Diagnostic accuracy and additional value of diffusion-weighted imaging for discrimination of malignant cervical lymph nodes in head and neck squamous cell carcinoma. Neuroradiology. 2009;51(3):183-92. doi: 10.1007/s00234-008-0487-2. [PubMed: 19137282].

20. Vandecaveye V, De Keyzer F, Vander Poorten V, Dirix P, Verbeken E, Nuyts S, et al. Head and neck squamous cell carcinoma: value of diffusion-weighted MR imaging for nodal staging. Radiology. 2009;251(1):134-46. doi: 10.1148/radiol.2511080128. [PubMed: 19251938]. 